\title{
IMPORTANCIA DE LAS DEFORMACIONES RECIENTES SOBRE LA ESTRUCTURACION GEOGRAFICA DE LA BAJA AMAZONIA PERUANA
}

(*) DUMONT JEAN FRANCOIS

\section{RESUMEN}

Los límites entre las zonas inundables y las zonas consolidadas no inundables estudiadas en áreas vecinas de dos estructuras mayores de la selva baja peruana (A rco de Iquitos y A rco de Contaya), se observan nítidos y controlados principalmente por deformaciones tectónicas ocurridas en el cuaternario. En varios casos, la ubicación actual de los grandes ríos de la cuenca amazónica parecen controlados estos movimientos recientes.

\section{INTRODUCCION}

En base a estudios de geología de superficie y de teledetección, realizados en la baja amazonía peruana, en el marco de un convenio entre la ORSTOM y el en el área de J enaro Herrera, aproximadamente $1-50 \mathrm{Km}$. al sur de la ciudad de Iquitos, se ha logrado establecer que las actividades tectónicas (fallas, flexuras) controlan la posición de las zonas consolidadas no inundables con respecto a las zonas inundables. Esta tectónica reciente interfiere con una dinámica fluvial poderosa, alteraciones pedológicas diversas y una vegetación muy diversificada, lo que implica la realización de estudios multidisciplinarios para su debida comprensión.

\section{MARCO GEOLOGICO: CUENCAS SUBSIIDENTES Y ARCOS NE OTECTONICOS}

A I Este del piedemonte morfo-tectónico de la faja subandina (Pardo 1982), la amazonía peruana comprende principalmente tres grandes cuencas geológicas cuaternarias, que son las siguientes de sur a norte (Fig. 1): la cuenca del río Madre de Dios, la cuenca del alto U cayali hasta Contamana y la cuenca del Nororiente peruano, correspondiente a la depresión donde se juntan los ríos, M arañón, Pastaza, Tigre y U cayali. La última es, de lejos, la más grande, pues drena el conjunto de las

(*) ORSTOM apartado 18-1209 Lima-Perú 
vertientes orientales de la cordillera andina entre uno (1) y trece (13) grados latitud sur.

Las cuencas del río Madre de Dios y del nororiente son independientes y drenan directamente hacia el exterior, en dirección al este 0 al noreste de los ríos nacidos el sector oriental de los A ndes.

En cambio, la cuenca del alto U cayali es una dependencia de la cuenca nororiente, con la que se junta por el norte. Las cuencas del alto U cayali y del nororiente son bordeadas al este por relieves poco elevados de gran extensi45n: el Arco de Contaya y el Arco de Iquitos.

Estas cuencas son, en parte, restos de las cuencas intracontinentales cenozoicas que acompañaron el levantamiento de los A ndes, acumulando importantes depósitos sedimentarios terciarios, Ilamados capas rojas, (Steimann 1930, Sanz 1974), en el piedemonte oriental de los relieves nuevamente formados.

La cuenca actual del nororiente está a continuación de la cuenca antigua de las capas rojas, que tiene sedimentación cuaternaria concordante (L aurent y Pardo 1975, Sanz 1974, Gutiérrez 1975). En cambio, una discontinuidad estratigráfica existe en las cuencas del alto U cayali y del Madre de Dios, donde los depósitos cuaternarios descansan en discordancia angular o de erosión por encima de las capas rojas (Campbell and Frailey 1984). Sin embargo, las zonas axiales de estas cuencas son actualmente subsidentes, lo que se manifiesta por la existencia de zonas inundadas muy extendidas en la cuenca nororiente y menos marcadamente, en las cuencas del alto U cayali y del Madre de Dios.

Los A rcos de Contaya y de Iquitos se sobreponen a zonas estructurales más antiguas, que fueron reactivadas durante las últimas fases tectónicas andinas, mayormente durante el plio-cuaternario. Las deformaciones recientes no son de gran amplitud, pero juegan un papel importante en la posición actual de los ríos y los límites entre las áreas consolidadas no inundables y las áreas inundables. Los proyectos actuales de investigación en geología y geomorfología, se enfocan principalmente hacia el estudio de los límites de las tierras inundables, que condicionan los aspectos físicos, humanos y económicos de la geografía de la amazonía peruana. 


\section{ESTUDIOS DE LOS LÍMITES DE LAS ZONAS CONSOLIDADAS NO INUNDABLES}

Estudios en detalle de límites entre las zonas consolidadas no inundables y las áreas inundables fueron iniciados en dos sectores: uno bordeando el Arco de Iquitos, en J enaro Herrera (Convenio ORSTOM -IIAP), el otro ubicado en la cuenca del Alto U cayali, desde A talaya hacia el A rco de Contaya (Convenio ORSTOM -IGP).

Los elementos que permiten caracterizar estos límites son cartográficos y litol6gicos; estudios preliminares en teledetección (Landsat, Slar y Spot); inventario y evaluación de las formaciones geológicas y sus características litológicas y eventualmente su datación. En algunos sectores, se pueden utilizar inventarios de los recursos naturales (ONERN: 1972, 1975, 1977 y 1978) y estudios pedológicos (V eillon 1986).

\section{AREA DE JENARO HERRERA}

L as áreas consolidadas no inundables del A rco de Iquitos están constituidas por depósitos de edad miocena a pliocuaternaria (formación Pebas: Ruegg y Rosensweig, 1949), formando un amplio entablamento poco elevado (promedio 30m.) pero fuertemente entallado por la red hidrográfica actual.

En el área de Jenaro Herrera (Fig. 2), las áreas consolidadas no inundables están delimitadas por alineamientos identificados por tele detección e interpretados como fallas normales. Las terrazas datadas con Carbono $14\left(\mathrm{C}^{-14}\right)(=40000 \mathrm{BP}$ y 13000 + 2090/- 1660 BP), a partir de grandes fragmentos de madera, están limitadas por estos alineamientos (Dumont et al. 1988), El estudio cinemático de las fallas normales que afectan los terrenos de la formación Ipururo, de edad pliocena hasta cuaternaria antigua, revela una fase tectónica extensiva de orientación noreste-sureste. Esta tectónica parece responsable de la formación de límites rectilíneos entre las áreas consolidadas no inundables y las áreas inundables, así cómo anomalías en la simetría de los meandros del U cayali y del Marañón, observables cerca de las localidades de Jenaro Herrera y de N auta.

\section{AREA DE ATALAYA}

En la selva central del Perú, el Arco de Contaya deslinda la divisoria entre el río U cayali y el río Purús. Constituye una zona deformada por plegamientos y fallas, con un relieve más elevado que domina en cerca de $300 \mathrm{~m}$. las cuencas del M adre de Dios y del Alto U cayali (Oppenheim 1975), y permite el afloramiento de la parte superior de la serie de las capas rojas. En la zona de Atalaya (Fig. 3), una terraza 


\section{8}

fluvial conglomerática descansa localmente en discordancia sobre las capas rojas; pero, sin embargo, ha sido deformada por el plegamiento del Arco. Se correlaciona lateralmente con las terrazas al tas de las quebradas de la faja subandina (formación Cañón, Dumont 1988), de edad pliocena. La fase tectónica comprensiva, de orientación noreste. sureste, que afecta la formación Cañón, puede ser relacionada con el plegamiento del arco de Contaya. U na de las consecuencias probables de esta fase tectónica es haber desviado hacia el norte el curso del U cayali, en dirección de la cuenca nororiente.

Los depósitos de la terraza antigua indican efectivamente que el curso del U cayali pasaba anteriormente más al este, y que podía cruzar la zona del Arco actual de Contaya hacia el río Y urúa.

El límite entre el Arco de Contaya y los terrenos inundables de la cuenca del A to U cayali, es imperceptible cuando la superficie estructural del plegamiento baja progresivamente hasta las tierras inundables. En cambio, al este de A talaya y en dirección del río U rubamba, el límite es un alineamiento correspondiente a una falla. A lo largo de este límite, una terraza baja limitada por falla (estrías de movimiento sobre cantos rodados) tiene una datación de $8990+750550$ años.

\section{CONCLUSION}

Los estudios geológicos realizados en la baja amazonía peruana revelan una tectónica cuaternaria de poca amplitud, pero influyendo fuertemente sobre la repartición de las áreas consolidadas no inundables y de las áreas inundables.

Los pasajes progresivos de una zona a la otra son raros y representan casos particulares, tales como el hundimiento de una superficie deformada 0 basculada. La influencia de la dinámica externa que se manifiesta mayormente a través de los ríos no modifica fundamentalmente la repartición de estas grandes unidades.

L a fortalece en cambio en amplios sectores, confinando las Ilanuras bajas de inundación entre los límites de alineamientos tectónicos y la complica en el detalle por la presencia de suelos hidromórficos dentro de las áreas consolidadas no inundables y de riveras inundables al nivel de las desembocaduras de las quebradas de las áreas no inundables en época de creciente. 


\section{REFERENCIAS BIBLIOGRAFICAS}

CAMPBELL, K. E., and FRAILEY, D., 1984.- Holocene flooding and species diversity in Southwestern A mazonia. Quaternary Research, 21, p. 369-375.

DUMONT, J. F., 1988.- Evolution néotectonique du piémont de la cordillere orientale des Andes centrales, région de San - Ramón. Geodynamique, en (prensa.)

DUMONT, J. F., LAMOTTE, S., y ÉOURNIER, M., 1988.- Neotectónica del A rco de Iquitos (Jenaro Herrera, Perú). Bol. Soc. Geol. del Perú, t. 77, p. 717.

GUTIERREZ, M. C., 1975.- Contribución al conocimiento micropaleontológico del Oriente Peruano. Bol. Soc. Geol. del Perú, t. 19, p. 25-52.

HAM C, K., HERRERA, L. J., 1963.- Role of subandean fault system in tectonics of Eastern Peru and Ecuador. Backbone of A merica, A mer. Pet. Geol., mem. 2, p. $47-61$.

LAURENT, H., et PARDO, A., 1975.- Ensayo de interpretación del basamento del Nororiente Peruano. Bol. Soc. Geol. del Perú, n. 45, p. 25-48.

ONERN, 1972.- Inventario, evaluación e integración de los recursos naturales de la zona de los ríos Inambari y Madre de Dios. Public. ONERN, Lima.

ONERN, 1975.- Inventario, evaluación e integración de los recursos naturales de la zona de A talaya. Public. ONERN, Lima.

ONERN, 1977.- U se of remote sensing systems evaluating the potential of the aguaje palm in the Peruvian J ungle. Public. ONERN, Lima.

ONERN, 1978.- Inventario, evaluación e integración de los recursos naturales de la zona de Pucallpa - A bujao - Public. ONERN, Lima.

OPPENHEIM, V., 1975.- The first (1944) geological exploration of the Upper A mazon V alley in Perú. Bol. Soc. Geol. del Perú, t. 45, p. 83 - 94.

PARDO, A., 1982.- Características estructurales de la faja subandina del norte del Perú. Simposio "Exploración petrolera en las cuencas subandinas de Venezuela, Colombia, Ecuador y Perú". 
RUEGO, W., y ROSENZWEIG, A., 1949.- Contribución a la geología de las formaciones modernas de Iquitos y de la amazonía superior. Sociedad Geológica del Perú, vol, jubilar, XXV A niversario, p. 1- 24.

SANZ, y. P. 1974.- Geología preliminar del área Tigre - Corrientes en el Oriente peruano. Bol. Soc. Geol. del Perú, t. XLIV, p. 106 - 127.

STEINM AINN, G., 1930.- Geología del Perú. Carl Winters Universitáts bichhandlung, $448 \mathrm{p}$.

VEILLON, L., 1986.- Rapport de Mission. Convenio IIA P/ORSTOM, Iquitos Lima. 


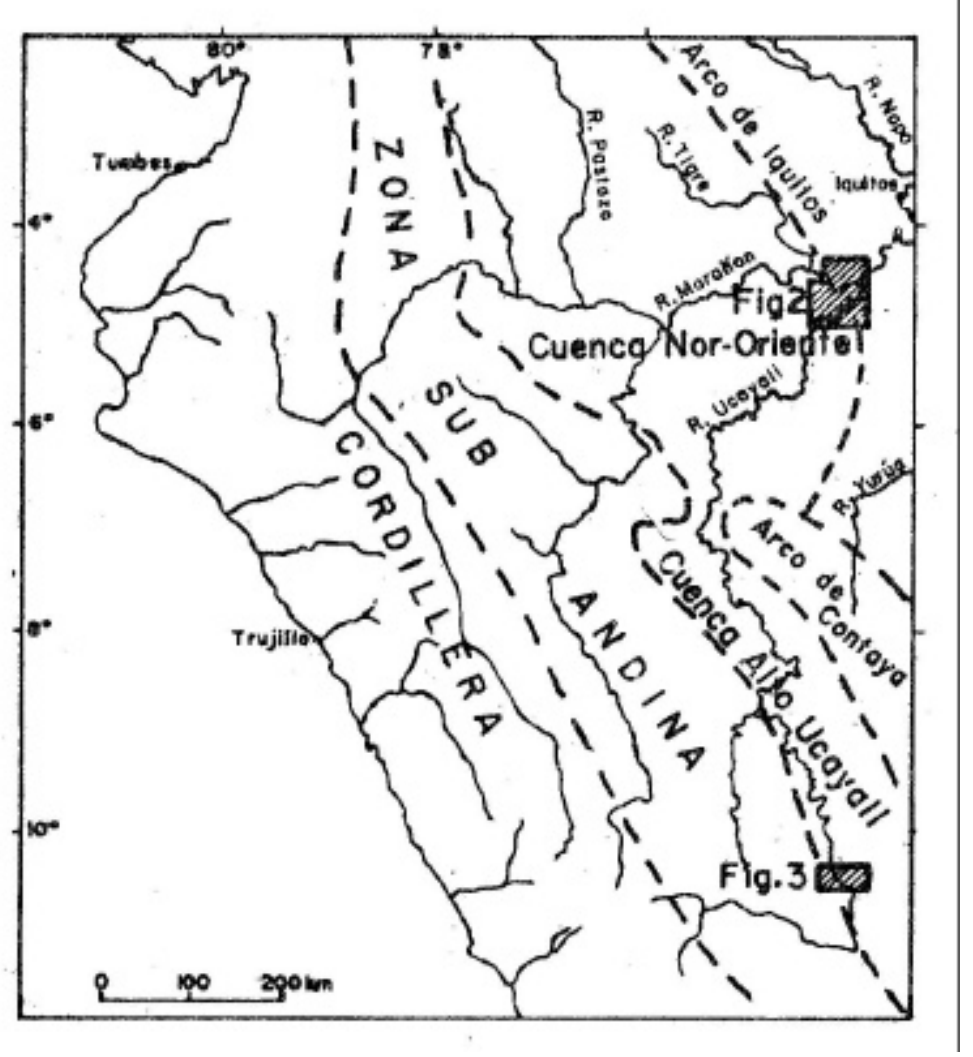

Fig. 1 Esquema de ubicación 


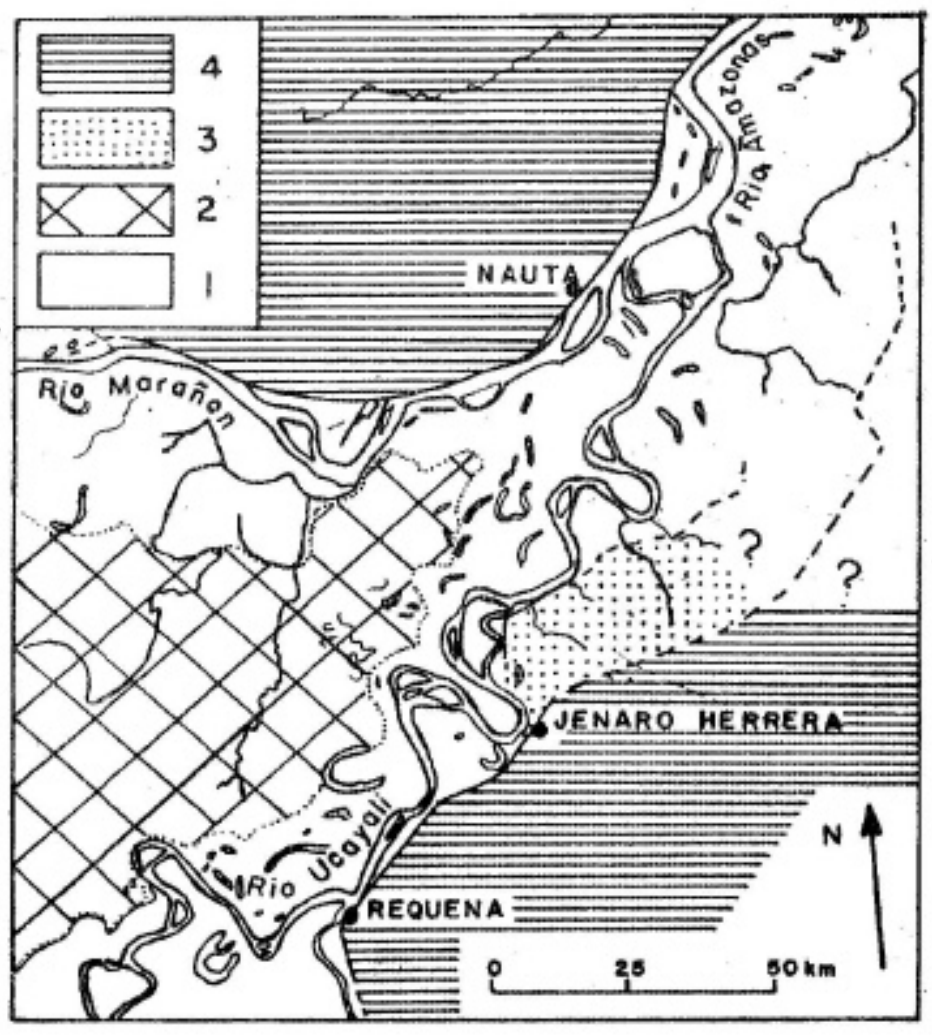

Fig. 2 Esquema estructural del Arco de Iquitos en la zona Nauta y J enaro Herrera (ubicada en la fig. 1)

\section{1: zonas inundables}

2: zonas inundables de aguas negras

3: terrazas cuaternarias recientes (40000 años y 13000 años)

4: " "area consolidada no inundable", de edad pliocena a cuaternaria antigua 


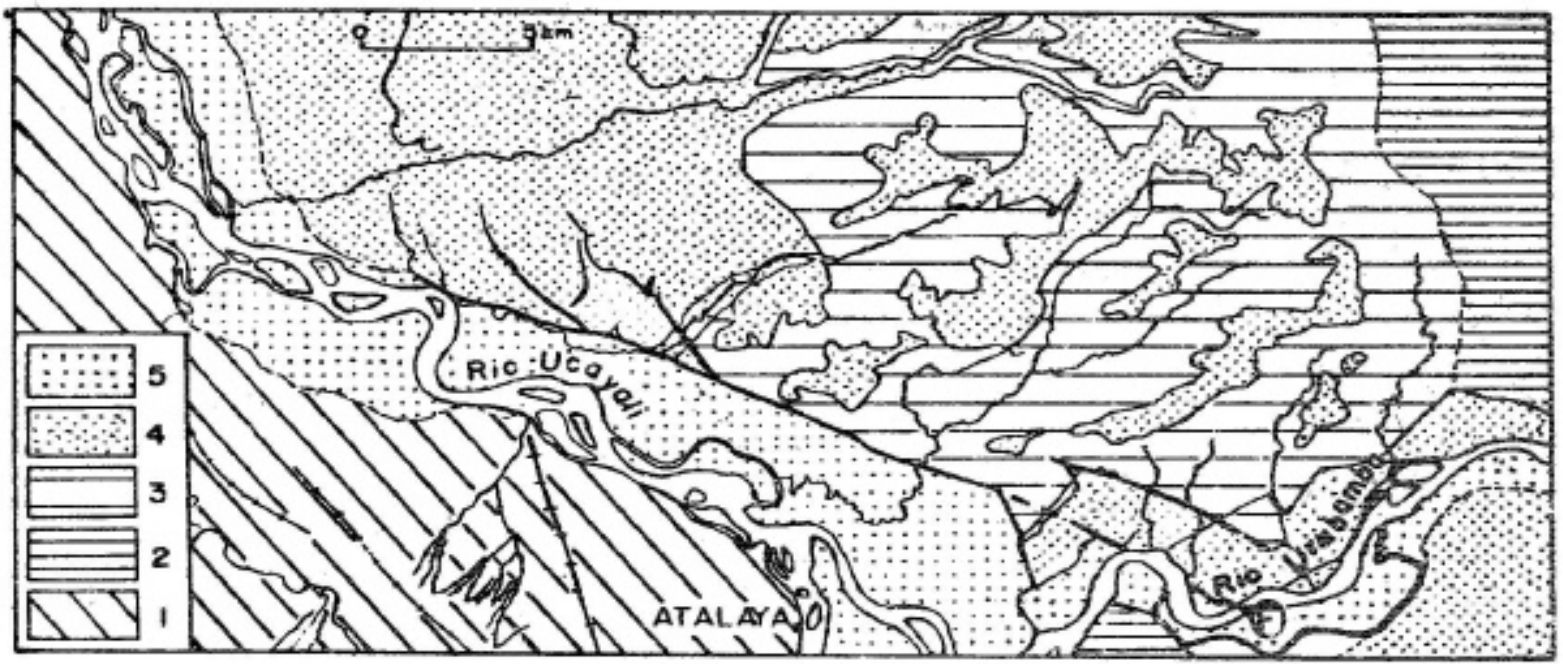

Fig. 3 E squema estructural de la zona de A talaya, (ubicada en la fig. 1).

1: selva alta de la zona subandina.

2: zonas profundamente erosionadas.

3: quebradas poco erosionadas.

4: superficie del plioceno superior/cuaternario antiguo.

5: terrazas bajas, totalmente 0 parcialmente inundables. 